\title{
Off-Site Population Radiological Dose and Risk Assessment for Potential Airborne Emissions from the Weldon Spring Site
}

by H.I. Avci, B.M. Biwer, and D.L. Blunt

rnvironmental Assessment and Information Sciences Division, Argonne National Laboratory, 9700 South Cass Avenue, Argonne, Illinois 60439

November 1992

Work sponsorer; 'jy United States Department of Energy, Weldon Spring Site Remedial Action Project 


\section{CONTENTS}

NOTATION $\ldots \ldots \ldots \ldots \ldots \ldots \ldots \ldots \ldots \ldots \ldots \ldots \ldots \ldots \ldots$

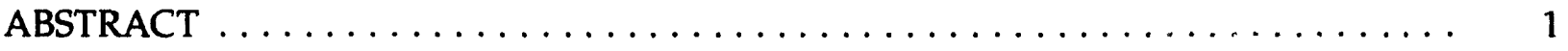

1 INTRODUCTION $\ldots \ldots \ldots \ldots \ldots \ldots \ldots \ldots \ldots \ldots \ldots \ldots \ldots \ldots \ldots \ldots$

2 METHODOLOGY $\ldots \ldots \ldots \ldots \ldots \ldots \ldots \ldots \ldots \ldots \ldots \ldots \ldots \ldots \ldots \ldots$

2.1 Environmental Transport . . . . . . . . . . . . . . . . . . 4

2.2 Estimation of Dose and Health Risk $\ldots \ldots \ldots \ldots \ldots \ldots \ldots \ldots \ldots$

3 INPUT PARAMETERS AND ASSUMPTIONS $\ldots \ldots \ldots \ldots \ldots \ldots \ldots$

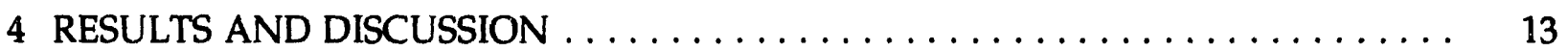

5 REASONABLE RADIUS OF MMPACT $\ldots \ldots \ldots \ldots \ldots \ldots \ldots \ldots \ldots \ldots$

6 REFERENCES ................................. 21

FIGURE

1 Location of the Weldon Spring Site $\ldots \ldots \ldots \ldots \ldots \ldots \ldots \ldots \ldots \ldots \ldots \ldots$

\section{TABLES}

1 CAP88-PC Input Parameters for Releases from the Weldon Spring Site . . . . . . 7

2 Distribution of the Population within a 50-Mile Radius of the Weldon Spring Site $\ldots \ldots \ldots \ldots \ldots \ldots \ldots \ldots \ldots \ldots \ldots \ldots \ldots$

3 Joint Wind Frequency Data File $\ldots \ldots \ldots \ldots \ldots \ldots \ldots \ldots \ldots \ldots$

4. Radionuclide Source Terms for the Proposed Remedial Action Alternatives at the Weldon Spring Site $\ldots \ldots \ldots \ldots \ldots \ldots \ldots \ldots \ldots$

5 Default CAP88-PC Input Parameters for Releases from the Weldon

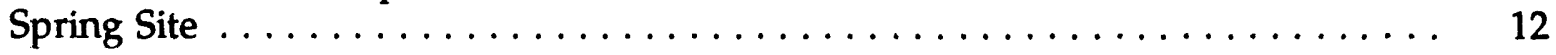

6 Estimated Population Doses and Health Effects from Atmospheric Releases during Cleanup Activities at the Weldon Spring Site: Chemical Stabilization/Solidification 


\section{TABLES (Cont.)}

7 Estimated Population Doses and Health Effects for Atmospheric Releases of 1 Curie of Radon-222 from the Weldon Spring Site . . . . . . . . . . . . .

8 Estimated Population Doses and Health Effects from Atmospheric Releases during Cleanup Activities at the Weldon Spring Site: Vitrification . . . . . . . 16

9 Distribution of Individual Dose Equivalent Rate with Distance and Direction around the Weldon Spring Site in 1998 for the Chemical Stabilization/Solidification Option, Excluding Radon-222

10 Distribution of Individual Dose Equivalent Rate with Distance and Direction around the Weldon Spring Site for 1 Curie

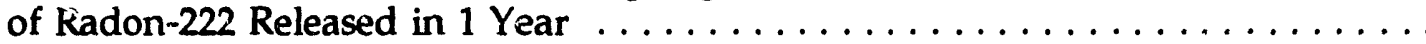

11 Estimated Radiological Impacts from Airborne Emissions for the Population within 3 Miles of the Weldon Spring Site . 


\section{NOTATION}

The following is a list of the acronyms, initialisms, and abbreviations (including units of measure) used in this document. Some acronyms used in tables or equations only are defined in the respective tables or equations.

\section{ACRONYMS, INITIALISMS, AND ABBREVIATIONS}

\begin{tabular}{|c|c|}
\hline BA & baseline assessment \\
\hline CDE & committed dose equivalent \\
\hline CEDE & committed effective dose equivalent \\
\hline CERCLA & $\begin{array}{l}\text { Comprehensive Environmental Response, Compensation, and Liability } \\
\text { Act of } 1980 \text {, as amended }\end{array}$ \\
\hline CFR & Code of Federal Regulations \\
\hline DOE & U.S. Department of Energy \\
\hline EDE & effective dose equivalent \\
\hline EPA & U.S. Environmental Protection Agency \\
\hline FS & feasibility study \\
\hline NCRP & National Council on Radiation Protection and Measurements \\
\hline NEPA & National Environmental Policy Act of 1969, as amended \\
\hline NESHAPS & National Emission Standards for Hazardous Air Pollutants \\
\hline NRC & U.S. Nuclear Regulatory Commission \\
\hline
\end{tabular}

\section{UNITS OF MEASURE}

$\begin{array}{ll}{ }^{\circ} \mathrm{C} & \text { degrees Celsius } \\ \mathrm{Ci} & \text { curie } \\ \mathrm{cm} & \text { centimeter } \\ \mathrm{cm}^{3} & \text { cubic centimeter } \\ \mathrm{d} & \text { day } \\ \mathrm{h} & \text { hour } \\ \mathrm{ha} & \text { hectare } \\ \mathrm{kg} & \text { kilogram } \\ \mathrm{km} & \text { kilometer } \\ \mathrm{km}^{2} & \text { square kilometer }\end{array}$

$\begin{array}{ll}\mathrm{L} & \text { liter } \\ \mathrm{m} & \text { meter } \\ \mathrm{m}^{2} & \text { square meter } \\ \mathrm{MeV} & \text { million electron volt } \\ \mathrm{mi} & \text { mile } \\ \mathrm{mrem} & \text { millirem } \\ \mathrm{rem} & \text { roentgen-equivalent man } \\ \mathrm{s} & \text { second } \\ \mathrm{yr} & \text { year }\end{array}$




\title{
OFF-SITE POPULATION RADIOLOGICAL DOSE AND RISK ASSESSMENT FOR POTENTIAL AIRBORNE EMISSIONS FROM THE WELDON SPRING SITE
}

by

\author{
H.I. Avci, B.M. Biwer, and D.L. Blunt
}

\begin{abstract}
Radiological doses and health risks to the population around the Weldon Spring site from potential airborne emissions during remedial action at the chemical plant area of the site have been assessed with the Clean Air Act Assessment Package-1988 computer code. Two treatment options are being considered for waste produced by site cleanup activities: chemical stabilization/solidification and vitrification. Over the entire cleanup period of 7 years, the collective dose received by the people who live within $80 \mathrm{~km}(50 \mathrm{mi})$ of the site (about 3 million persons) is estimated to be about 34 person-rem for the chemical stabilization/ solidification option and 32 person-rem for the vitrification option. By comparison, the same population is expected to receive about $6 \times 10^{6}$ person-rem from natural background radiation during that time. If only the population within a reasonable radius of impact is considered (about 10,700 persons live within $5 \mathrm{~km}$ [ $3 \mathrm{mi}$ ] of the site), the remedial action activities are estimated to result in about 5 person-rem over the entire cleanup period; the same population is expected to receive about 20,000 person-rem from natural background radiation during that time. Because the doses are low, no cancers or genetic effects are expected to occur among the population around the Weldon Spring site as a result of exposures resulting from potential radioactive releases to the atmosphere during remediation of the chemical plant area.
\end{abstract}

\section{INTRODUCTION}

The Weldon Spring site is located in St. Charles County, Missouri, about $48 \mathrm{~km}$ (30 mi) west of St. Louis (Figure 1). The site became contaminated as a result of processing and disposal activities that took place from the 1940s through the 1960s, and it is listed on the National Priorities List of the U.S. Environmental Protection Agency (EPA). The U.S. Department of Energy (DOE) is responsible for cleanup activities at the site under its Environmental Restoration and Waste Management Program.

The Weldon Spring site consists of two noncontiguous areas: an 88-ha (217-acre) chemical plant area and a 3.6-ha (9-acre) limestone quarry. The chemical plant area is about $3.2 \mathrm{~km}(2 \mathrm{mi})$ southwest of the junction of Missouri (State) Route 94 and U.S. 40/61, and the 


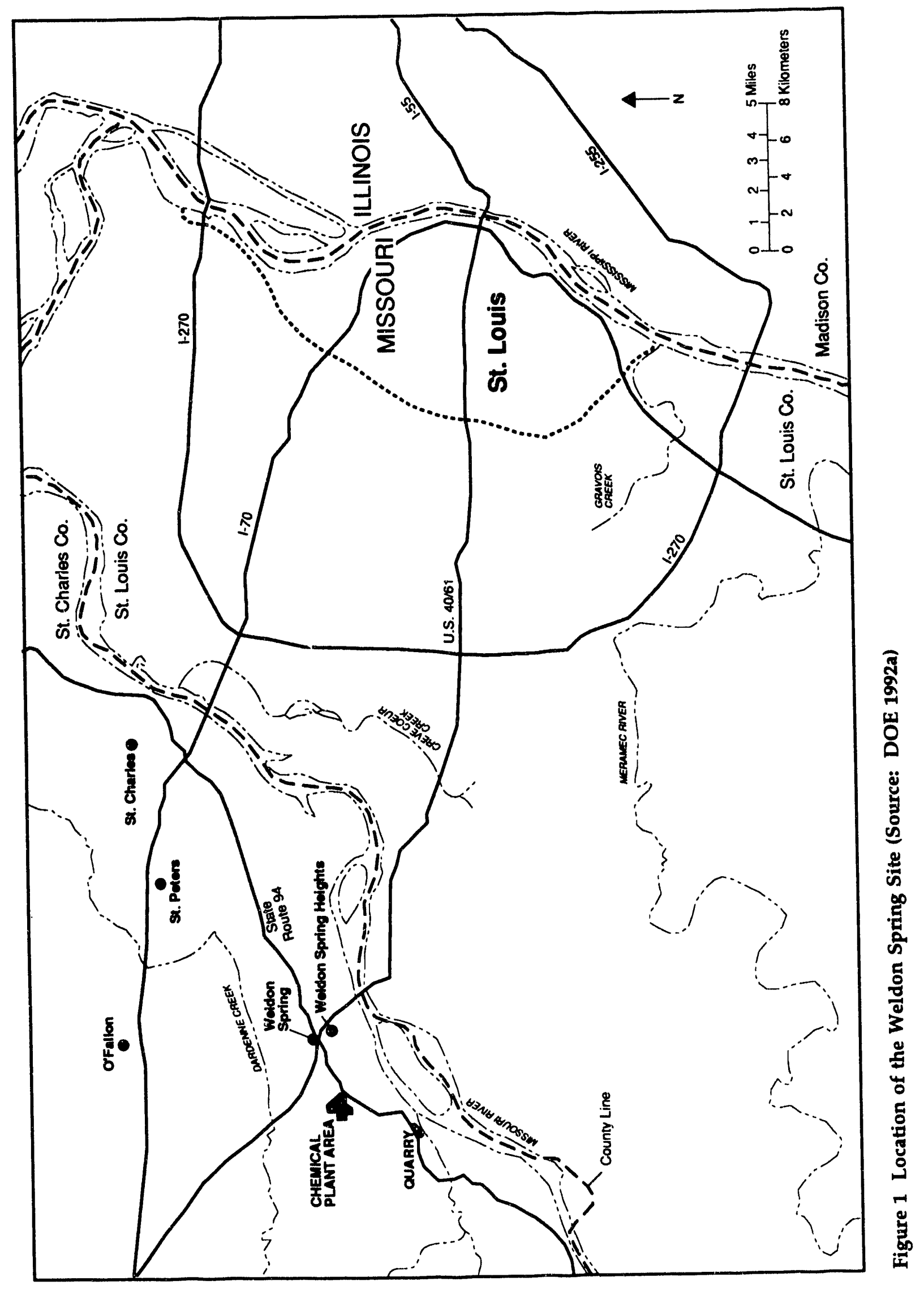


quarry is about $6.4 \mathrm{~km}(4 \mathrm{mi})$ south-southwest of the chemical plant area. Both locations are accessible from State Route 94 and are fenced and closed to the, :ublic. Explosives were produced at the chemical plant during the 1940s, and uranium and thorium materials were processed during the 1950s and 1960s. During the latter operational period, waste slurries at the chemical plant area were piped to four retention ponds, referred to as raffinate pits; various solid wastes (i.e., process residues and decontamination material that included soil, rubble, metal debris, and equipment) were disposed of in the quarry between 1942 and 1969.

In accordance with the requirements of the Comprehensive Environmental Response, Compensation, and Liability Act (CERCLA), as amended, a remedial investigation/feasibility study (RI/FS) process has been performed for the chemical plant area of the Weldon Spring site. The RI/FS package compiises four documents: an RI report (DOE 1992d), a baseline assessment (BA) (DOE 1992a), an FS report (DOE 1992b), and a proposed plan (DOE 1992c). These documents were prepared in accordance with CERCLA requirements, incorporating values associated with the National Environmental Policy Act (NEPA). As part of the FS, the radiological impacts to the population near the site from potential airborne releases during the remedial action period have been assessed. The results of this assessment are summarized in the FS. This report describes the calcuiations associated with these analyses.

Radiological doses were calculated with Version 1.0 of the CAP88-PC personal computer code (EPA 1992), and the risks to human health were calculated by multiplying the doses obtained from CAP88-PC with risk factors used by the EPA (1989a). The assessment covered the two proposed options for waste treatment: chemical stabilization/solidification and vitrification. Potential doses and health risks were calculated for the oft site population within an $80-\mathrm{km}(50-\mathrm{mi})$ radius of the site. From the results of these calculations, a radius of reasonable impact was inferred and the health risks to the population within this radius were calculated.

The methods used to estimate the collective radiological doses to members of the general public for this report differ somewhat from those used to estimate the doses to specific individual receptors in the BA and FS. The radiological doses presented herein are based on the methodology provided in the CAP88-PC computer code, whereas the doses in the BA and FS are largely based on the methodology given in the Risk Assessment Guidanci for Superfund (EPA $1989 \mathrm{~b})$. However, these methodological differences are small and do not affect the results and conclusions given in the various documents developed for the Weldon Spring site. The population doses presented in this document are based on 1990 census data. 


\section{METHODOI.OGY}

The CAP88-PC computer code is a personal-computer version of the Clean Air Act Assessment Package-1988 (CAP-88) (Beres 1990), which is one of the codes specified by the EPA in Title 40, Code of Federal Regulations, Part 61, Subpart H (40 CFR Part 61, Subpart H), for demonstrating compliance with the National Emission Standards for Hazardous Air Pollutants (NESHAPs). The CAP88-PC computer code was developed by the EPA's Office of Radiation Programs (EPA 1992). This code comprises a set of subprograms, databases, and associated utility programs that implement a mathematical model for assessing dose and health risk from radionuclide emissions to the atmosphere. The code evaluates the environmental transport of radionuclides released to the atmosphere and estimates health impacts from the following pathways: inhalation, ingestion, and external gamma irradiation from air immersion and the ground surface. Results are tabulated for individuals residing at specified locations and for regional populations.

\subsection{ENVIRONMENTAL TRANSPORT}

The CAP88-PC program models environmental transport in a manner similar to that documented in its predecessor model, AIRDOS-PRA (Moore et al. 1979). The CAP88-PC program uses a modified Gaussian plume equation to estimate the average dispersion of radionuclides released from as many as six sources. The sources may be either elevated stacks or uniform area sources, such as a pile of uranium mill tailings. Plume rises can be calculated by assuming either a momentum-driven or buoyancy-driven plume. The assessment areas encompass a circular grid within a given radius around the modeled facility.

The CAP88-PC program computes radionuclide concentrations in air, rates of deposition on ground surfaces, concentrations in food, and intake rates for humans from inhalation of air and ingestion of food produced in the assessment area. The radionuclide concentrations in produce, leafy vegetables, milk, and meat consumed by humans are estimated by coupling the output of the atmospheric transport models with the terrestrial food chain models given in Regulatory Guide 1.109 of the U.S. Nuclear Regulatory Commission (NRC 1977).

\subsection{ESTIMATION OF DOSE AND HEALTH RISK}

Dose conversion factors in CAP88-PC are provided for intakes of radionuclides by ingestion and inhalation and also for external gamma irradiation from air immersion and the ground surface. Factors are further broken down by particle size, solubility class, and digestion transfer factors. These factors are generated with the RADRISK computer program (Dunning et al. 1980; Begovich et al. 1981).

In CAP88-PC, dose and health risk are estimated by the DARTAB program (Begovich et al. 1981), which combines the inhalation and ingestion intake rates and the air and ground surface concentrations with the dose and risk factors from the RADRISK database. The effective 
dose equivalent is calculated with the weighting factors given in Publication 26 of the International Commission on Radiological Protection (1977). The CAP88-PC code lists the dose and risk to individuals residing at the specified locations and to the collective population. Doses and risks are further tabulated as a function of radionuclide, pathway, location, and organ. More inforntation concerning CAP88-PC and its methodology is given in the User's Guide for CAP88-PC (EPA 1992) and the reports of Beres (1990) and the Radiation Shielding Information Center (1987).

For this assessment, the CAP88-PC code was used to calculate the doses received by selected hypothetical off-site individuals and the collective population. The risks in terms of cancer induction and genetic effects among the exposed population were calculated as follows. The cancer risk was calculated by multiplying the collective committed effective dose equivalent (CEDE) by the risk factor of $6 \times 10^{-4} /$ person-rem (EPA 1989a). The genetic effects, defined as the number of severe physical or mental defects in the offspring of exposed persons, were calculated by multiplying the sum of the collective committed dose equivalent (CDE) to gonads and the collective external effective dose equivalent (EDE) by the risk factor of $2.6 \times 10^{-4}$ /person-rem (EPA 1989a). 


\section{INPUT PARAMETERS AND ASSUMPTIONS}

Site-specific data used as input to the CAP88-PC code are given in Table 1 . The $80-\mathrm{km}$ $(50-\mathrm{mi})$ off-site population distribution and wind frequency data used are provided in Tables 2 and 3. The average year and maximum year atmospheric releases of radionuclides (source terms) were calculated for each of the two waste treatment options. The average year releases for each radionuclide were determined by dividing the total release of radionuclides by 7 , the number of years estimated for completion of cleanup activities. The radionuclide source terms used in the assessment are listed in Table 4. Only the principal radionuclides in the uranium-238, uranium-235, and thorium-232 decay series have been included in the source terms (see the discussion in Section 2.3.2.1 of the BA [DOE 1992a]). Analyses performed with the CAP-88 code (before the CAP88-PC code was available) for the assessment reported in this document showed that the short-lived decay products in these decay series (except for the daughters of radon-222, which are accounted for in both CAP-88 and CAP88-PC) contributed less than $10 \%$ to the total CEDE.

The input parameters other than the radionuclide source terms are listed in Table 5; these parameters are CAP88-PC default values. A more complete description of CAP88-PC input parameters and the default values assigned to these parameters in the code are provided in EPA (1992). 
TABLE 1 CAP88-PC Input Parameters for Releases

from the Weldon Spring Site

\begin{tabular}{|c|c|}
\hline Parameter & Value \\
\hline \multicolumn{2}{|l|}{ Radionuclide release data } \\
\hline Stack (release) height & $10 \mathrm{~m}$ \\
\hline Period of long-term buildup activity in the soil & 40 years \\
\hline Release rate of radionuclides to the atmosphere & See Table 4 \\
\hline \multicolumn{2}{|l|}{ Meteorological data } \\
\hline Rainfall rate & $95 \mathrm{~cm} / \mathrm{yr}$ \\
\hline Average temperature & $13^{\circ} \mathrm{C}$ \\
\hline Height of tropospheric mixing layer & $900 \mathrm{~m}$ \\
\hline Height for wind speed measurements & $10 \mathrm{~m}$ \\
\hline Joint wind frequency data & See Table 3 \\
\hline \multicolumn{2}{|l|}{ Receptor data } \\
\hline Inhalation rate of humans & $8.3 \times 10^{5} \mathrm{~cm}^{3} / \mathrm{h}$ \\
\hline Total population & $2,922,000$ \\
\hline Population distribution & See Table 2 \\
\hline \multicolumn{2}{|l|}{ Rate of ingestion by humans ${ }^{a}$} \\
\hline Produce & $105 \mathrm{~kg} / \mathrm{yr}$ \\
\hline Milk & $140 \mathrm{~L} / \mathrm{yr}$ \\
\hline Meat & $35 \mathrm{~kg} / \mathrm{yr}$ \\
\hline Leafy vegetables & $14 \mathrm{~kg} / \mathrm{yr}$ \\
\hline \multicolumn{2}{|l|}{ Source of food for individual's diet ${ }^{a, b}$} \\
\hline \multicolumn{2}{|l|}{ Milk } \\
\hline At location of individual & $75 \%$ \\
\hline Within assessment area & 0 \\
\hline Outside assessment area & $25 \%$ \\
\hline \multicolumn{2}{|l|}{ Meat } \\
\hline At location of individual & $75 \%$ \\
\hline Within assessment area & 0 \\
\hline Outside assessment area & $25 \%$ \\
\hline \multicolumn{2}{|l|}{ Vegetables } \\
\hline At location of individual & $40 \%$ \\
\hline Within assessment area & 0 \\
\hline Outside assessment area & $60 \%$ \\
\hline
\end{tabular}

a As recommended by the EPA (1991). Leafy vegetables were assumed to constitute $20 \%$ of the EPA-recommended vegetable component (NRC 1977).

b Assessment area is defined as an $80-\mathrm{km}(50-\mathrm{mi})$ radius around the release point. 
TABLE 2 Distribution of the Population within a 50-Mile Radius of the Weldon Spring Site

\begin{tabular}{|c|c|c|c|c|c|c|c|c|c|c|}
\hline \multirow{2}{*}{$\begin{array}{c}\text { Wind } \\
\text { Direction }\end{array}$} & \multicolumn{10}{|c|}{ Population per Sector ${ }^{a}$} \\
\hline & $0-1$ & $1-2$ & $2-3$ & $3-4$ & $4-5$ & $5-10$ & $10-20$ & $20-30$ & $30-40$ & $40-50$ \\
\hline $\mathrm{N}$ & 73 & 220 & 367 & 513 & 660 & 5,501 & 11,619 & 3,361 & 3,793 & 3,794 \\
\hline NNW & 73 & 220 & 367 & 513 & 660 & 5,501 & 10,554 & 4,626 & 5,984 & 4,388 \\
\hline NW & 73 & 220 & 367 & 513 & 660 & 5,501 & 13,106 & 4,623 & 5,973 & 4,397 \\
\hline WNW & 73 & 220 & 367 & 513 & 660 & 5,501 & 7316 & 4,423 & 3,906 & 3,832 \\
\hline W & 73 & 220 & 367 & 313 & 660 & 5,501 & 5,461 & 4,618 & 5,912 & 4,296 \\
\hline WSW & 73 & 220 & 367 & 513 & 660 & 5,501 & 7,258 & 7,980 & 9,500 & 4,811 \\
\hline SW & 73 & 220 & 367 & 513 & 660 & 5,501 & 10,498 & 8,475 & 11,865 & 9,286 \\
\hline SSW & 73 & 220 & 367 & 513 & 660 & 4,309 & 5,176 & 8,475 & 9,564 & 4,711 \\
\hline S & 73 & 220 & 367 & 720 & 2,026 & 14,787 & 35,168 & 18,399 & 22,880 & 11,689 \\
\hline SSE & 73 & 220 & 369 & 2,352 & 3,575 & 29,791 & 72,099 & 25,586 & 35,034 & 38,143 \\
\hline SE & 73 & 220 & 530 & 2,780 & 3,575 & 29,791 & 103,546 & 63,510 & 9,027 & 10,584 \\
\hline ESE & 73 & 220 & 371 & 2,123 & 3,573 & 29,791 & 119,163 & 497,757 & 45,254 & 66,709 \\
\hline E & 73 & 220 & 367 & 513 & 992 & 10,951 & 115,628 & 663,537 & 48,873 & 61,633 \\
\hline ENE & 73 & 220 & 367 & 513 & 660 & 5,501 & 69,893 & 150,550 & 68,722 & 48,746 \\
\hline NE & 73 & 220 & 367 & 513 & 660 & 5,501 & 20,635 & 19,380 & 13,961 & 9,436 \\
\hline NNE & 73 & 220 & 367 & 513 & 660 & 5,501 & 7,300 & 4,268 & 5,951 & 5,142 \\
\hline
\end{tabular}

a Each sector is expressed as a range of miles : $: 0 \mathrm{~m}$ the release point for the population within $80 \mathrm{~km}$ $(50 \mathrm{mi})$ from the site. The population file ...nsists of 10 radial sectors in each of 16 directions from the release point. Each entry is the popula ticn ior a given sector in a particular direction away from the release point. All entries are based on 1990 census data and assume that the population is uniformly distributed within each county. This approach overestimates the actual population living within $5 \mathrm{~km}(3 \mathrm{mi})$ of the site. 
TABLE 3 Joint Wind Frequency Data File

\begin{tabular}{|c|c|c|c|c|c|c|c|}
\hline \multirow{2}{*}{$\begin{array}{l}\text { Wind } \\
\text { Direction }\end{array}$} & \multirow{2}{*}{$\begin{array}{c}\text { Stability } \\
\text { Class }\end{array}$} & \multicolumn{6}{|c|}{ Wuiu Frequency at Wind Speed Bin } \\
\hline & & 1.5 knots & 5.0 knots & 8.5 knots & 13.5 knots & 19.0 knots & 23.0 knots \\
\hline $\mathbf{N}$ & $\mathbf{A}$ & 0.00054 & 0.00068 & 0.00000 & 0.00000 & 0.00000 & 0.00000 \\
\hline NNE & A & 0.00066 & 0.00057 & 0.00000 & 0.00000 & 0.00000 & 0.00000 \\
\hline NE & A & 0.00032 & 0.00091 & 0.00000 & 0.00000 & 0.00000 & 0.00000 \\
\hline ENE & A & 0.00037 & 0.00160 & 0.00000 & 0.00000 & 0.00000 & 0.00000 \\
\hline $\mathrm{E}$ & A & 0.00033 & 0.00114 & 0.00000 & 0.00000 & 0.00000 & 0.00000 \\
\hline ESE & A & 0.00010 & 0.00126 & 0.00000 & 0.00000 & 0.00000 & 0.00000 \\
\hline SE & A & 0.00005 & 0.00068 & 0.00000 & 0.00000 & 0.00000 & 0.00000 \\
\hline SSE & A & 0.00033 & 0.00114 & 0.00000 & 0.00000 & 0.00000 & 0.00000 \\
\hline $\mathbf{S}$ & A & 0.00034 & 0.00126 & 0.00000 & 0.00000 & 0.00000 & 0.00000 \\
\hline SSW & A & 0.00003 & 0.00046 & 0.00000 & 0.00000 & 0.00000 & 0.00000 \\
\hline SW & A & 0.00006 & 0.00080 & 0.00000 & 0.00000 & 0.00000 & 0.00000 \\
\hline WSW & $\mathbf{A}$ & 0.00003 & 0.00034 & 0.00000 & 0.00000 & 0.00000 & 0.00000 \\
\hline$W$ & A & 0.00018 & 0.00080 & 0.00000 & 0.00000 & 0.00000 & 0.00000 \\
\hline WNW & A & 0.00014 & 0.00023 & 0.00000 & 0.00000 & 0.00000 & 0.00000 \\
\hline NW & $\mathbf{A}$ & 0.00025 & 0.00011 & 0.00000 & 0.00000 & 0.00000 & 0.00000 \\
\hline NNW & A & 0.00025 & 0.00011 & 0.00000 & 0.00000 & 0.00000 & 0.00000 \\
\hline $\mathbf{N}$ & B & 0.00130 & 0.00285 & 0.00000 & 0.00000 & 0.00000 & 0.00000 \\
\hline NNE & B & 0.00062 & 0.00183 & 0.00000 & 0.00000 & 0.00000 & 0.00000 \\
\hline NE & B & 0.00061 & 0.00171 & 0.00000 & 0.00000 & 0.00000 & 0.00000 \\
\hline ENE & B & 0.00123 & 0.00194 & 0.00000 & 0.00000 & 0.00000 & 0.00000 \\
\hline $\mathrm{E}$ & B & 0.00101 & 0.00400 & 0.00000 & 0.00000 & 0.00000 & 0.00000 \\
\hline ESE & B & 0.00031 & 0.00091 & 0.00000 & 0.00000 & 0.00000 & 0.00000 \\
\hline SE & B & 0.00043 & 0.00091 & 0.00000 & 0.00000 & 0.00000 & 0.00000 \\
\hline SSE & B & 0.00051 & 0.00205 & 0.00000 & 0.00000 & 0.00000 & 0.00000 \\
\hline $\mathbf{S}$ & B & 0.00069 & 0.00468 & 0.00000 & 0.00000 & 0.00000 & 0.00000 \\
\hline SSW & B & 0.00049 & 0.00171 & 0.00000 & 0.00000 & 0.00000 & 0.00000 \\
\hline SW & B & 0.00032 & 0.00103 & 0.00000 & 0.00000 & 0.00000 & 0.00000 \\
\hline WSW & B & 0.00014 & 0.00194 & 0.00000 & 0.00000 & 0.00000 & 0.00000 \\
\hline$w$ & B & 0.00065 & 0.00228 & 0.00000 & 0.00000 & 0.00000 & 0.00000 \\
\hline WNW & B & 0.00036 & 0.00171 & 0.00000 & 0.00000 & 0.00000 & 0.00000 \\
\hline NW & B & 0.00037 & 0.00183 & 0.00000 & 0.00000 & 0.00000 & 0.00000 \\
\hline NNW & B & 0.00068 & 0.00103 & 0.00000 & 0.00000 & 0.00000 & 0.00000 \\
\hline $\mathbf{N}$ & C & 0.00069 & 0.00434 & 0.00274 & 0.00000 & 0.00000 & 0.00000 \\
\hline NNE & $C$ & 0.00059 & 0.00228 & 0.00046 & 0.00000 & 0.00000 & 0.00000 \\
\hline NE & C & 0.00112 & 0.00331 & 0.00103 & 0.00011 & 0.00000 & 0.00000 \\
\hline ENE & C & 0.00087 & 0.00308 & 0.00034 & 0.00000 & 0.00000 & 0.00000 \\
\hline $\mathrm{E}$ & C & 0.00153 & 0.00445 & 0.00068 & 0.00000 & 0.00000 & 0.00000 \\
\hline ESE & C & 0.00105 & 0.00194 & 0.00091 & 0.00000 & 0.00000 & 0.00000 \\
\hline SE & C & 0.00160 & 0.00342 & 0.00091 & 0.00000 & 0.00000 & 0.00000 \\
\hline SSE & C & 0.00158 & 0.00537 & 0.00171 & 0.00000 & 0.00000 & 0.00000 \\
\hline $\mathrm{S}$ & C & 0.00174 & 0.00868 & 0.00594 & 0.00000 & 0.00000 & 0.00000 \\
\hline SSW & C & 0.00066 & 0.00365 & 0.00194 & 0.00000 & 0.00000 & 0.00000 \\
\hline SW & C & 0.00080 & 0.00411 & 0.00126 & 0.00000 & 0.00000 & 0.00000 \\
\hline WSW & C & 0.00085 & 0.00514 & 0.00240 & 0.00000 & 0.00000 & 0.00000 \\
\hline $\mathbf{W}$ & $\mathrm{C}$ & 0.00091 & 0.00400 & 0.00342 & 0.00000 & 0.00000 & 0.00000 \\
\hline WNW & $C$ & 0.00062 & 0.00285 & 0.00240 & 0.00000 & 0.00000 & 0.00000 \\
\hline NW & $C$ & 0.00069 & 0.00194 & 0.00183 & 0.00000 & 0.00000 & 0.00000 \\
\hline NNW & $C$ & 0.00126 & 0.00365 & 0.00251 & 0.00000 & 0.00000 & 0.00000 \\
\hline $\mathbf{N}$ & D & 0.00562 & 0.01518 & 0.01119 & 0.00068 & 0.00000 & 0.00000 \\
\hline NNE & D & 0.00413 & 0.01142 & 0.00639 & 0.00011 & 0.00000 & 0.00000 \\
\hline NE & $\mathrm{D}$ & 0.00460 & 0.01119 & 0.00571 & 0.00034 & 0.00000 & 0.00000 \\
\hline
\end{tabular}


TABLE 3 (Cont.)

\begin{tabular}{|c|c|c|c|c|c|c|c|}
\hline \multirow{2}{*}{$\begin{array}{c}\text { Wind } \\
\text { Direction }\end{array}$} & \multirow{2}{*}{$\begin{array}{c}\text { Stability } \\
\text { Class }\end{array}$} & \multicolumn{6}{|c|}{ Wind Frequency at Wind Speed Bin } \\
\hline & & 1.5 knots & 5.0 knots & 8.5 knots & 13.5 knots & 19.0 knots & 23.0 knots \\
\hline ENE & D & 0.00392 & 0.00685 & 0.00126 & 0.00023 & 0.00000 & 0.00000 \\
\hline $\mathbf{E}$ & D & 0.00649 & 0.01358 & 0.00194 & 0.00080 & 0.00000 & 0.00000 \\
\hline ESE & D & 0.00479 & 0.00879 & 0.00228 & 0.00000 & 0.00011 & 0.00000 \\
\hline SE & D & 0.00749 & 0.01221 & 0.00285 & 0.00000 & 0.00000 & 0.00000 \\
\hline SSE & D & 0.00768 & 0.02500 & 0.00731 & 0.00011 & 0.00000 & 0000 \\
\hline 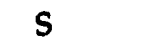 & D & 0.01022 & 0.04669 & 0.02249 & 0.00342 & 0.00000 & 0.00000 \\
\hline SSW & D & 0.00573 & 0.02683 & 0.01655 & 0.00297 & 0.00000 & 0.00000 \\
\hline SW & D & 0.00493 & 0.00902 & 0.00605 & 0.00240 & 0.00000 & 0.00000 \\
\hline WsW & D & 0.00329 & 0.00993 & 0.00445 & 0.00354 & 0.00011 & 0.00000 \\
\hline W & D & 0.00473 & 0.01632 & 0.01507 & 0.00685 & 0.00023 & 0.00000 \\
\hline WNW & D & 0.00578 & 0.01233 & 0.01541 & 0.00753 & 0.00046 & 0.00000 \\
\hline NW & $\bar{D}$ & 0.00437 & 0.01484 & 0.01495 & 0.00616 & 0.00091 & 0.00000 \\
\hline NNW & D & 0.00493 & 0.01404 & 0.01244 & 0.00285 & 0.00000 & 0.00000 \\
\hline $\mathbf{N}$ & E & 0.00000 & 0.01176 & 0.00320 & 0.00000 & 0.00000 & 0.00000 \\
\hline NNE & E & 0.00000 & 0.00491 & 0.00114 & 0.00000 & 0.00000 & 0.00000 \\
\hline NE & E & 0.00000 & 0.00491 & 0.00068 & 0.00000 & 0.00000 & 0.00000 \\
\hline ENE & $E$ & 0.00000 & 0.00194 & 0.00000 & 0.00000 & 0.00000 & 0.00000 \\
\hline $\mathbf{E}$ & E & 0.00000 & 0.00411 & 0.00023 & 0.00000 & 0.00000 & 0.00000 \\
\hline ESE & E & 0.00000 & 0.00491 & 0.00023 & 0.00000 & 0.00000 & 0.00000 \\
\hline SE & E & 0.00000 & 0.01084 & 0.00080 & 0.00000 & 0.00000 & 0.00000 \\
\hline SSE & E & 0.00000 & 0.01301 & 0.00114 & 0.00000 & 0.00000 & 0.00000 \\
\hline$s$ & E & 0.00000 & 0.02705 & 0.00582 & 0.00000 & 0.00000 & 0.00000 \\
\hline SSW & $\bar{E}$ & 0.00000 & 0.01564 & 0.00365 & 0.00000 & 0.00000 & 0.00000 \\
\hline SW & E & 0.00000 & 0.00811 & 0.00114 & 0.00000 & 0.00000 & 0.00000 \\
\hline WSW & E & 0.00000 & 0.00479 & 0.00160 & 0.00000 & 0.00000 & 0.00000 \\
\hline $\mathbf{w}$ & E & 0.00000 & 0.00947 & 0.00274 & 0.00000 & 0.00000 & 0.00000 \\
\hline WNW & $\overline{\mathrm{E}}$ & 0.00000 & 0.00788 & 0.00297 & 0.00000 & 0.00000 & 0.00000 \\
\hline NW & E & 0.00000 & 0.01005 & 0.00354 & 0.00000 & 0.00000 & $0.000 \mathrm{no}$ \\
\hline NNW & E & 0.00000 & 0.00776 & 0.00183 & 0.00000 & 0.00000 & 0.00000 \\
\hline $\mathbf{N}$ & $\mathbf{F}$ & 0.00501 & 0.00011 & 0.00000 & 0.00000 & 0.00000 & 0.00000 \\
\hline NNE & F & 0.00396 & 0.00023 & 0.00000 & 0.00000 & 0.00000 & 0.00000 \\
\hline NE & F & 0.00505 & 0.00023 & 0.00000 & 0.00000 & 0.00000 & 0.00000 \\
\hline ENE & F & 0.00392 & 0.00011 & 0.00000 & 0.00000 & 0.00000 & 0.00000 \\
\hline E & F & 0.00673 & 0.00057 & 0.00000 & 0.00000 & 0.00000 & 0.00000 \\
\hline ESE & F & 0.00563 & 0.00011 & 0.00000 & 0.00000 & 0.00000 & 0.00000 \\
\hline SE & $\mathrm{F}$ & 0.01018 & 0.00068 & 0.00000 & 0.00000 & 0.00000 & 0.00000 \\
\hline SSE & $F$ & 0.00606 & 0.00046 & 0.00000 & 0.00000 & 0.00000 & 0.00000 \\
\hline $\mathbf{S}$ & $\mathrm{F}$ & 0.01316 & 0.00205 & 0.00000 & 0.00000 & 0.00000 & 0.00000 \\
\hline SSW & F & 0.00952 & 0.00228 & 0.00000 & 0.00000 & 0.00000 & 0.00000 \\
\hline SW & F & 0.00587 & 0.00034 & 0.00000 & 0.00000 & 0.00000 & 0.00000 \\
\hline WSW & F & 0.00563 & 0.00011 & 0.00000 & 0.00000 & 0.00000 & 0.00000 \\
\hline W & F & 0.01290 & 0.00046 & 0.00000 & 0.00000 & 0.00000 & 0.00000 \\
\hline WNW & F & 0.00752 & 0.00148 & 0.00000 & 0.00000 & 0.00000 & 0.00000 \\
\hline NW & F & 0.00901 & 0.00046 & 0.00000 & 0.00000 & 0.00000 & 0.00000 \\
\hline NNW & $\mathbf{F}$ & 0.00548 & 0.00011 & 0.00000 & 0.00000 & 0.00000 & 0.00000 \\
\hline
\end{tabular}

2 This data file contains stability array (STAR) data that show the frequencies of occurrence of the wind blowing from a particular direction (column 1), at a particular stability (classes A to F, column 2), at a particular speed. For each direction and each stability class, the probability of occurrence is listed for each of six wind speed bins (columns 3 to 8 ). The wind speed bins correspond to 1.5, 5.0, 8.5, 13.5, 19.0 , and 23.0 knots $(0.772,2.572,4.372,6.945,9.774$, and $11.832 \mathrm{~m} / \mathrm{s})$, respectively. 
TABLE 4 Radionuclide Source Terms for the Proposed Remedial Action Alternatives at the Weldon Spring Sitea

\begin{tabular}{|c|c|c|c|c|}
\hline \multirow[b]{2}{*}{ Radionuclide } & \multicolumn{2}{|c|}{$\begin{array}{c}\text { Source Terms for } \\
\text { Chemical Stabilization/Solidification } \\
(\mathrm{Ci} / \mathrm{yr})\end{array}$} & \multicolumn{2}{|c|}{$\begin{array}{l}\text { Source Terms for Vitrification } \\
\qquad(\mathrm{Ci} / \mathrm{yr})\end{array}$} \\
\hline & Average Year & Maximum Year ${ }^{b}$ & Average Year & Maximum Year \\
\hline Actinium-227 & $2.7 \times 10^{-5}$ & $4.7 \times 10^{-5}$ & $1.5 \times 10^{-5}$ & $2.7 \times 10^{-5}$ \\
\hline Lead-210 & $4.1 \times 10^{-5}$ & $8.2 \times 10^{-5}$ & $1.0 \times 10^{-3}$ & $1.5 \times 10^{-3}$ \\
\hline Protactinium-231 & $3.6 \times 10^{-5}$ & $6.1 \times 10^{-5}$ & $2.1 \times 10^{-5}$ & $3.5 \times 10^{-5}$ \\
\hline Radium-226 & $3.5 \times 10^{-5}$ & $6.3 \times 10^{-5}$ & $2.3 \times 10^{-5}$ & $4.0 \times 10^{-5}$ \\
\hline Radium-228 & $1.6 \times 10^{-5}$ & $3.0 \times 10^{-5}$ & $1.1 \times 10^{-5}$ & $2.1 \times 10^{-5}$ \\
\hline Radon-222 & $3.5 \times 10^{1}$ & $5.0 \times 10^{1}$ & $5.0 \times 10^{1}$ & $6.6 \times 10^{1}$ \\
\hline Thorium-230 & $1.2 \times 10^{-3}$ & $2.1 \times 10^{-3}$ & $6.7 \times 10^{-4}$ & $1.2 \times 10^{-3}$ \\
\hline Thorium-232 & $4.1 \times 10^{-6}$ & $6.5 \times 10^{-6}$ & $3.5 \times 10^{-6}$ & $5.3 \times 10^{-6}$ \\
\hline Uranium-235 & $3.8 \times 10^{-6}$ & $6.0 \times 10^{-6}$ & $2.8 \times 10^{-6}$ & $4.2 \times 10^{-6}$ \\
\hline Uranium-238 & $8.2 \times 10^{-5}$ & $1.3 \times 10^{-4}$ & $6.1 \times 10^{-5}$ & $8.9 \times 10^{-5}$ \\
\hline
\end{tabular}

a The radiological doses associated with the other radionuclides in the uranium-238, thorium-232, and uranium-235 radioactive decay series were calculated on the basis of the results of the radiological source term analysis. See related discussion in Sections 2.3.2.1 and 4.1 of the BA (DOE 1992a).

b The maximum year is 1998 for all radionuclides except radon-222. For radon-222, the maximum year is 1994 under chemical stabilization/solidification and 1995 under vitrification. 
TABLE 5 Default CAP88-PC Input Parameters for Releases from the Weldon Spring Site

\begin{tabular}{|c|c|}
\hline Input Parameter & Value \\
\hline $\begin{array}{l}\text { Agricultural productivity by unit area } \\
\text { Grass-cow-milk-humans pathway } \\
\text { Produce or leafy vegetables ingested by humans }\end{array}$ & $\begin{array}{l}0.280 \mathrm{~kg} / \mathrm{m}^{2} \\
0.716 \mathrm{~kg} / \mathrm{m}^{2}\end{array}$ \\
\hline Fraction of year animals graze on pasture & 0.40 \\
\hline $\begin{array}{l}\text { Fraction of daily ieed that is pasture grass when anirnal grazes } \\
\text { on pasture }\end{array}$ & 0.43 \\
\hline $\begin{array}{l}\text { Period of exposure during growing season } \\
\text { Pasture grass } \\
\text { Crops or leafy vegetables }\end{array}$ & $\begin{array}{l}720 \text { hours } \\
1,440 \text { hours }\end{array}$ \\
\hline $\begin{array}{l}\text { Fallout interception fraction } \\
\text { Vegetables } \\
\text { Pasture }\end{array}$ & $\begin{array}{l}0.20 \\
0.57\end{array}$ \\
\hline Removal rate constant for physical loss by weathering & $2.9 \times 10^{-3}$ per hour \\
\hline Buildup time for radionuclides deposited on ground and water & $1.5 \times 10^{4}$ days \\
\hline $\begin{array}{l}\text { Consumption rate of contaminated feed or forage by an animal } \\
\text { (dry weight) }\end{array}$ & $15.6 \mathrm{~kg} / \mathrm{d}$ \\
\hline Milk production of cow & $11.0 \mathrm{~L} / \mathrm{d}$ \\
\hline Transport time from animal feed-milk-humans & 2.0 days \\
\hline $\begin{array}{l}\text { Time delay } \\
\text { Ingestion of pasture grass by animals } \\
\text { Ingestion of stored feed by animals } \\
\text { Ingestion of leafy vegetables by humans } \\
\text { Ingestion of produce by humans }\end{array}$ & $\begin{array}{l}0 \text { hours } \\
2,160 \text { hours } \\
336 \text { hours } \\
340 \text { hours }\end{array}$ \\
\hline $\begin{array}{l}\text { Fraction of radioactivity retained on leafy vegetables and produce } \\
\text { after washing }\end{array}$ & 0.5 \\
\hline Average time from slaughter of animal to consumption of meat & 20 days \\
\hline Muscle mass of animal at slaughter & $200 \mathrm{~kg}$ \\
\hline Fraction of animal herd slaughtered per day & $3.8 \times 10^{-3}$ \\
\hline Beef cattle density & $3.43 \times 10^{-1} / \mathrm{km}^{2}$ \\
\hline Milk cattle density & $1.89 \times 10^{-2} / \mathrm{km}^{2}$ \\
\hline Land fraction cultivated for vegetable crops & $8.14 \times 10^{-3}$ \\
\hline
\end{tabular}




\section{RESULTS AND DISCUSSION}

The estimated radiological impacts to the population within $80 \mathrm{~km}(50 \mathrm{mi})$ of the Weldon Spring site, assuming chemical stabilization/solidification of the waste, are presented in Table 6 . The results are presented both excluding and including the impacts associated with radon-222 releases. The estimated doses and health effects associated with radon-222 are presented in Table 7; these estimates are based on the release of $1 \mathrm{Ci}$ of radon-222 in 1 year. For the chemical stabilization/solidification treatment option, the average annual radon-222 release is estimated to be $35 \mathrm{Ci}$. The maximum amount of radon-222 released in any year is estimated to be $50 \mathrm{Ci}$. Table 6 also shows the estimated annual doses to the same population (about 3 million persons) from natural background radiation. The estimated dose from cleanup activities is less than $0.001 \%$ of the dose received from the natural background.

The contributions to the collective 50-year CEDE by exposure pathway, the doses associated with the two radionuclides contributing most of the dose (radon-222 and thorium-230), and the doses to two specific organs (the thyroid and the gonads) are given in Table 6. The health effects in terms of cancer induction and genetic effects among the exposed population were estimated with EPA risk factors (EPA 1989a), as described in Section 2.2.

The estimated radiological doses and health effects among the population within $80 \mathrm{~km}$ $(50 \mathrm{mi})$ of the Weldon Spring site as a result of cleanup activities are given in Table 8 for the vitrification option. During vitrification, the average and maximum radon-222 releases are estimated to be 50 and $66 \mathrm{Ci} / \mathrm{yr}$, respectively. The total collective CEDE estimated for that population is slightly less for the vitrification option than the chemical stabilization/solidification option. However, either option would produce impacts that are much less than those that would result from natural background radiation during the same time period. 
TABLE 7 Estimated Population Doses and Health Effects for Atmospheric Releases of 1 Curie of Radon-222 from the Weldon Spring Site ${ }^{a}$

\begin{tabular}{|c|c|c|}
\hline Parameter & 1 Year & $\begin{array}{l}\text { During 7-Year } \\
\text { Cleanup Period }\end{array}$ \\
\hline CEDE $^{b}$ (person-rem) & $5.0 \times 10^{-2}$ & $3.5 \times 10^{-1}$ \\
\hline External exposure & $4.5 \times 10^{-6}$ & $3.2 \times 10^{-5}$ \\
\hline Inhalation & $5.0 \times 10^{-2}$ & $3.5 \times 10^{-1}$ \\
\hline Ingestion & 0.0 & 0.0 \\
\hline Person-working level ${ }^{c}$ & $1.5 \times 10^{-2}$ & $1.1 \times 10^{-1}$ \\
\hline Thyroid $\mathrm{CDE}^{\mathrm{d}}$ (person-rem) & $4.8 \times 10^{-2}$ & $3.4 \times 10^{-1}$ \\
\hline Gonads CDE (person-rem) & $4.9 \times 10^{-2}$ & $3.4 \times 10^{-1}$ \\
\hline Cancer incidents ${ }^{\mathbf{e}}$ & $3 \times 10^{-5}$ & $2 \times 10^{-4}$ \\
\hline Genetic effects ${ }^{\mathrm{f}}$ & $1 \times 10^{-5}$ & $9 \times 10^{-5}$ \\
\hline
\end{tabular}

a Estimated for the population of about 2,922,000 within an $80-\mathrm{km}(50-\mathrm{mi})$ radius of the site.

b $\mathrm{CEDE}=$ committed effective dose equivalent. Also listed by pathway (external exposure, inhalation, ingestion).

c One working level is defined as any combination of short-lived decay products of radon-222 in $1 \mathrm{~L}$ of air that will release $1.3 \times 10^{5} \mathrm{MeV}$ of alpha particle energy. Person-working level is the integrated sum of the radon decay product concentrations to which the population within the $80-\mathrm{km}(50-\mathrm{mi})$ radius of the site is exposed.

d $\mathrm{CDE}=$ committed dose equivalent.

e Calculated by multiplying the CEDE by the risk factor of $6 \times 10^{-4} /$ person-rem (EPA 1989a). Rounded to one significant figure.

f Genetic effects are defined as the calculated number of severe physical or mental defects in the offspring of exposed persons. Calculated by multiplying the sum of the gonads CDE and the external dose by the risk factor of $2.6 \times 10^{-4} /$ person-rem (EPA 1989a). Rounded to one significant figure. 
TABLE 8 Estimated Population Doses and Health Effects from Atmospheric Releases during Cleanup Activities at the Weldon Spring Site: Vitrification ${ }^{\mathrm{a}}$

\begin{tabular}{|c|c|c|c|c|c|}
\hline \multirow[b]{2}{*}{ Parameter } & \multicolumn{3}{|c|}{ Excluding Radon-222 } & \multicolumn{2}{|c|}{ Including Radon-222 } \\
\hline & $\begin{array}{l}\text { Average } \\
\text { Year }\end{array}$ & $\underset{\text { Year }^{b}}{\text { Maximum }}$ & $\begin{array}{l}\text { During 7-Year } \\
\text { Cleanup Period }\end{array}$ & $\begin{array}{l}\text { Average } \\
\text { Year }\end{array}$ & $\begin{array}{l}\text { During 7-Year } \\
\text { Cleanup Period }\end{array}$ \\
\hline CEDE $^{c}$ (person-rem) & 2.0 & 3.5 & $1.4 \times 10^{1}$ & 4.5 & $3.2 \times 10^{1}$ \\
\hline $\begin{array}{l}\text { External exposure } \\
\text { Inhalation } \\
\text { Ingestion }\end{array}$ & $\begin{array}{l}6.3 \times 10^{-4} \\
1.8 \\
1.8 \times 10^{-1}\end{array}$ & $\begin{array}{l}9.9 \times 10^{-4} \\
3.2 \\
2.7 \times 10^{-1}\end{array}$ & $\begin{array}{l}4.4 \times 10^{-3} \\
1.3 \times 10^{1} \\
1.3\end{array}$ & $\begin{array}{l}8.6 \times 10^{-4} \\
4.3 \\
1.8 \times 10^{-1}\end{array}$ & $\begin{array}{l}6.0 \times 10^{-3} \\
3.0 \times 10^{1} \\
1.3\end{array}$ \\
\hline $\begin{array}{l}\text { Thorium-230 } \\
\text { Radon-222 }\end{array}$ & ${ }^{1.5} \mathrm{NA}^{\mathrm{d}}$ & $\stackrel{2.6}{N A}$ & $\begin{array}{c}1.1 \times 10^{1} \\
\mathrm{NA}\end{array}$ & $\begin{array}{l}1.5 \\
2.5\end{array}$ & $\begin{array}{l}1.1 \times 10^{1} \\
1.8 \times 10^{1}\end{array}$ \\
\hline $\begin{array}{l}\text { Thyroid } \mathrm{CDE}^{e} \\
\text { (person-rem) }\end{array}$ & $3.0 \times 10^{-2}$ & $4.7 \times 10^{-2}$ & $2.1 \times 10^{-1}$ & 2.4 & $1.7 \times 10^{1}$ \\
\hline $\begin{array}{l}\text { Gonads CDE } \\
\text { (person-rem) }\end{array}$ & $3.7 \times 10^{-2}$ & $5.9 \times 10^{-2}$ & $2.6 \times 10^{-1}$ & 2.5 & $1.7 \times 10^{1}$ \\
\hline Cancer incidents $\mathrm{f}$ & $1 \times 10^{-3}$ & $2 \times 10^{-3}$ & $8 \times 10^{-3}$ & $3 \times 10^{-3}$ & $2 \times 10^{-2}$ \\
\hline Genetic effects8 & $1 \times 10^{-5}$ & $2 \times 10^{-5}$ & $7 \times 10^{-5}$ & $7 \times 10^{-4}$ & $5 \times 10^{-3}$ \\
\hline $\begin{array}{l}\text { Natural background } \\
\text { (person-rem) }\end{array}$ & $3 \times 10^{5}$ & $3 \times 10^{5}$ & $2 \times 10^{6}$ & $9 \times 10^{5}$ & $6 \times 10^{6}$ \\
\hline
\end{tabular}

a Estimated for the population of about $2,922,000$ within an $80-\mathrm{km}(50-\mathrm{mi})$ radius of the site.

b Maximum year is 1998, according to current plans.

c $\mathrm{CEDE}=$ committed effective dose equivalent. Also listed by pathway (external exposure, inhalation, ingestion) and by radionuclide (only the major contributors).

d $\mathrm{NA}=$ not applicable.

e $\mathrm{CDE}=$ conis atted dose equivalent.

f Calculated by multiplying the CEDE by the risk factor of $6 \times 10^{-4} /$ person-rem (EPA 1989a). Rounded to one significant figure.

8 Genetic effects are defined as the calculated number of severe physical or mental defects in the offspring of exposed persons. Calculated by multiplying the sum of the gonads CDE and the external dose by the risk factor of $2.6 \times 10^{-4} /$ person-rem (EPA 1989a). Rounded to one significant figure.

h Assumed to be $0.1 \mathrm{rem} / \mathrm{yr}$ per person excluding radon and $0.3 \mathrm{rem} / \mathrm{yr}$ per person including radon, which are the national averages (NCRP 1987a). The background dose is assumed to be the same for both the average year and the maximum year. 


\section{REASONABLE RADIUS OF IMPACT}

One measure of the reasonable radius of impact around the Weldon Spring site could be the distance at which an individual would receive a relatively small fraction of the maximum individual dose from cleanup activities. The variation of an individual's dose equivalent rate (the weighted sum of organ doses from all pathways) with distance and direction under the chemical stabilization/solidification option is given in Table 9 for all radionuclides except radon-222 and in Table 10 for radon-222. The doses in Table 9 are for 1998, the year when all radionuclides except radon-222 are projected to be released in the largest amount for any year during operations, according to current plans. The doses in Table 10 were calculated for $1 \mathrm{Ci}$ of radon-222 released in 1 year. To obtain the doses from the release of $X$ curies of radon-222 in 1 year, the values in the table have to be multiplied by $X$. Note that an individual receives successively less dose in all directions as the individual moves farther away from the site.

The results presented in Tables 9 and 10 indicate that the highest dose would be about $1.3 \mathrm{mrem} / \mathrm{yr}$ for a hypothetical individual living at the site boundary to the north. The estimated dose to a hypothetical individual at the site boundary averaged over all directions is about $0.58 \mathrm{mrem} / \mathrm{yr}$. An individual located $5 \mathrm{~km}(3 \mathrm{mi})$ from the site would receive about $5 \%$ of the dose received by an individual at the site boundary. This distance, $5 \mathrm{~km}(3 \mathrm{mi})$, is taken to be the reasonable radius of impact for the site. The value of $5 \mathrm{~km}(3 \mathrm{mi})$ is conservative because the NCRP (1987b) has recommended that population assessments be limited to those individuals receiving a dose in excess of $1 \mathrm{mrem} / \mathrm{yr}$ from site releases.

About 10,700 persons reside within $5 \mathrm{~km}(3 \mathrm{mi})$ of the Weldon Spring site. (This estimate is based on 1990 census data and the average population density of St. Charles and St. Louis counties. The number of people actually residing within this radius is significantly lower. Thus, the population doses calculated for the potentially impacted population are overestimated.) The estimated collective radiological impacts for these individuals from cleanup activities are listed in Table 11. The highest population dose associated with these activities in any 1 year is about 1.2 person-rem, which is about $0.04 \%$ of the dose these same individuals would receive from natural background radiation in an average year. 


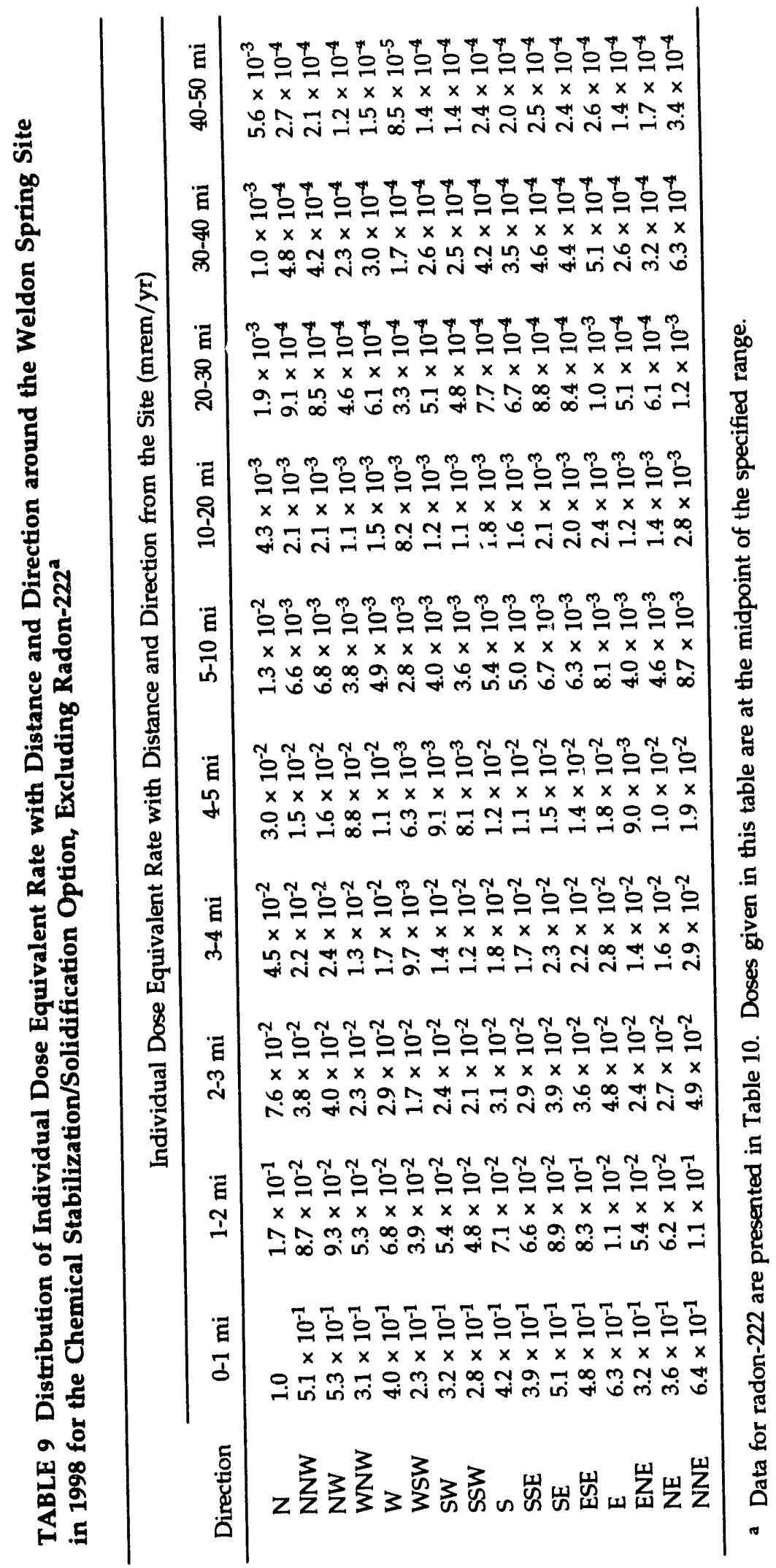




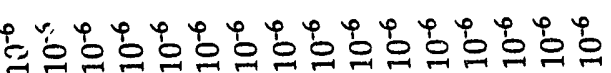
$\times \times \times \times \times \times \times \times \times \times \times \times \times \times \times \times$

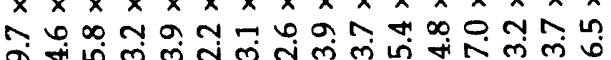

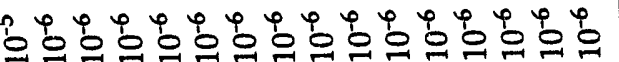
$\times \times \times \times \times \times \times \times \times \times \times \times \times \times \times \times$

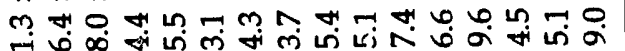
पुक्ष

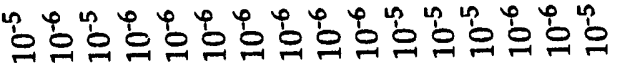

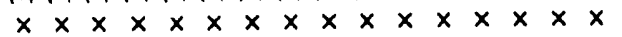
নై

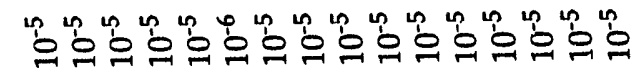

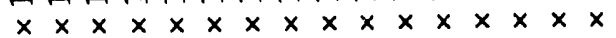
б. mं

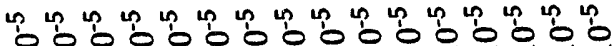

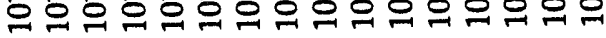
$\times \times \times \times \times \times \times \times \times \times \times \times \times \times \times \times$ m

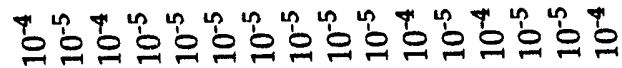
$\times \times \times \times \times \times \times \times \times \times \times \times \times \times \times x$ ब궁ำ

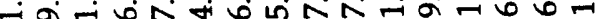

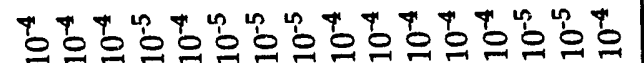
$\times \times \times \times \times \times \times \times \times \times \times \times \times \times \times x$

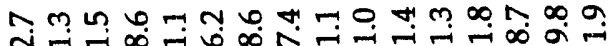

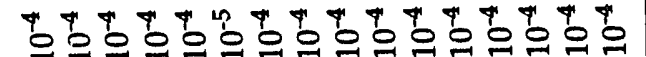
$\times \times \times \times \times \times \times \times \times \times \times \times \times \times \times \times$ भં

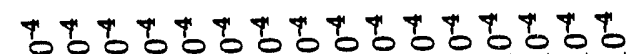

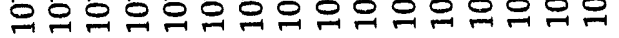

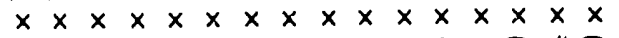

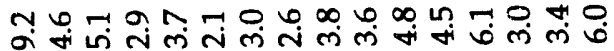

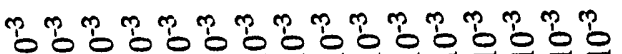
$\times \times \times \times \times \times \times \times \times \times \times \times \times \times \times \times$ 官 
TABLE 11 Estimated Radiological Impacts from Airborne Emissions for the Population within 3 Miles of the Weldon Spring Site ${ }^{a}$

\begin{tabular}{|c|c|c|c|}
\hline $\begin{array}{c}\text { Treatment Option/ } \\
\text { Parameter }\end{array}$ & Average Year & Maximum Year ${ }^{b}$ & $\begin{array}{l}\text { During 7-Year } \\
\text { Cleanup Period }\end{array}$ \\
\hline \multicolumn{4}{|l|}{ Chemical stabilization/solidification } \\
\hline $\begin{array}{l}\mathrm{CEDE}^{\mathrm{c}} \text { (person-rem) } \\
\text { Cancer incidents }^{\mathrm{d}}\end{array}$ & $\begin{array}{c}7.8 \times 10^{-1} \\
5 \times 10^{-4}\end{array}$ & $7 \times 10^{-4}$ & $\begin{array}{c}5.5 \\
3 \times 10^{-3}\end{array}$ \\
\hline \multicolumn{4}{|l|}{ Vitrification } \\
\hline $\begin{array}{l}\mathrm{CEDE}^{c} \text { (person-rem) } \\
\text { Cancer incidents } \mathrm{d}\end{array}$ & $\begin{array}{c}6.6 \times 10^{-1} \\
4 \times 10^{-4}\end{array}$ & $\begin{array}{c}9.3 \times 10^{-1} \\
6 \times 10^{-4}\end{array}$ & $\begin{array}{c}4.6 \\
3 \times 10^{-3}\end{array}$ \\
\hline Natural background ${ }^{e}$ (person-rem) & $3 \times 10^{3}$ & $3 \times 10^{3}$ & $2 \times 10^{4}$ \\
\hline
\end{tabular}

a Estimated for the population of about 10,700 within $5 \mathrm{~km}(3 \mathrm{mi})$ of the site.

b Maximum year is 1998, according to current plans.

c $\mathrm{CEDE}=$ committed effective dose equivalent.

d Calculated by multiplying the CEDE by the risk factor of $6 \times 10^{-4} /$ person-rem (EPA 1989a). Rounded to one significant figure.

e Assumed to be $0.3 \mathrm{rem} / \mathrm{yr}$ per person, which is the national average (NCRP 1987a). The background dose is assumed to be the same for both the average year and the maximum year. 


\section{REFERENCES}

Begovich, C.L., et al., 1981, DARTAB, A Program to Combine Airborne Radionuclide Environmental Exposure Data with Dosimetric Health Effects Data to Generate Tabulations of Predicted Health Impact, ORNL-5692/DE81030434, Oak Ridge National Laboratory, Oak Ridge, Tenn., Aug.

Beres, D.A., 1990, The Clean Air Act Assessment Package-1988 (CAP-88) - A Dose and Risk Assessment Methodology for Radionuclide Emissions to Air, Volumes 1, 2, and 3, SC\&A, Inc., McLean, Va.

DOE: see U.S. Department of Energy.

Dunning D.E., Jr., et al., 1980, A Combined Methodology for Estimating Dose Rates and Health Effects from Exposure to Radioactive Pollutants, ORNL/TM-7105, Oak Ridge National Laboratory, Oak Ridge, Tenn., March.

EPA: see U.S. Environmental Protection Ágency.

International Commission on Radiological Protection, 1977, Recommendations of the International Commission on Radiological Protection, ICRP Publication 26, Annals of the ICRP, 1(1), Pergamon Press, Oxford, U.K.

Moore, R.E., et al., 1979, AIRDOS-EPA: A Computerized Methodology for Estimating Environmental Concentrations and Dose to Man from Airborne Releases of Radionuclides, ORNL-5532, Oak Ridge National Laboratory, Oak Ridge, Tenn., June.

National Council on Radiation Protection and Measurements, 1987a, Exposure of the Population in the United States and Canada from Natural Background Radiation, NCRP Report No. 94, Bethesda, Md., Dec. 30.

National Council on Radiation Protection and Measurements, 1987b, Recommendations on Limits for Exposure to lonizing Radiation, NCRP Report No. 91, Bethesda, Md., June 1.

NCRP: see National Council on Radiation Protection and Measurements.

NRC: see U.S. Nuclear Regulatory Commission.

Radiation Shielding Information Center, 1987, CAAC - Code System for Implementation of Atmospheric Dispersion Assessment Required by the Clean Air Act, CCC-476, Radiation Shielding Information Center, Oak Ridge National Laboratory, Oak Ridge, Tenn.

U.S. Department of Energy, 1992a, Baseline Assessment for the Chemical Plant Area of the Weldon Spring Site, DOE/OR/21548-091, prepared by Argonne National Laboratory, Environmental Assessment and Information Sciences Division, Argonne, Ill., for U.S. Department of Energy, Oak Ridge Field Office, Weldon Spring Site Remedial Action Project, St. Charles, Mo., Nov. 
U.S. Department of Energy, 1992b, Feasibility Study for Remedial Action at the Chemicr! Plant Area of the Weldon Spring Site, DOE/OR/21548-148, Vols. I-II, prepared by Argonne National Laboratory, Environmental Assessment and Information Sciences Division, Argonne, III.. for U.S. Department of Energy, Oak Ridge Field Office, Weldon Spring Site Remedial Action Project, St. Charies, Mo., Nov.

U.S. Department of Energy, 1992c, Proposed Plan for Remedial Action at the Chemical Plant Area of the Weldon Spring Site, DOE/OR/21548-160, prepared by Argonne National Laboratory, Environmental Assessment and Information Sciences Division, Argonne, Ill., for U.S. Department of Energy, Oak Ridge Field Office, Weldon Spring Site Remedial Action Project, St. Charles, Mo., Nov.

U.S. Department of Energy, 1992d, Remedial Investigation for the Chemical Plant Area of the Weldon Spring Site, DOE/OR/21548-074, Rev. 0, Vols. I-II, prepared by MK-Ferguson Company and Jacobs Engineering Group, Weldon Spring, Mo., for U.S. Department of Energy, Oak Ridge Field Office, Weldon Spring Site Remedial Action Project, St. Charles, Mo., Nov.

U.S. Environmental Protection Agency, 1989a, Risk Assessment Methodology, Environmental Impact Statement for NESHAPS Radionuclides, Volume 1, Background Information Document, EPA/520/ 1-89/005, Office of Radiation Programs, Washington, D.C., Sept. 7.

U.S. Environmental Protection Agency, 1989b, Risk Assessment Guidance for Superfund, Volume I: Human Health Evaluation Manual (Part A), EPA/540/1-89/002, Interim Final, Office of Emergency and Remedial Response, Washington, D.C., Dec.

U.S. Environmental Protection Agency, 1991, Human Health Evaluation Manual, Supplemental Guidance: "Standard Default Exposure Factors," OSWER Directive 9285.6-03, March 25.

U.S. Environmental Protection Agency, 1992, User's Guide for CAP88-PC, Version 1.0, 402-B-92-001, Office of Radiation Programs, Las Vegas, Nev., March.

U.S. Nuclear Regulatory Commission, 1977, Calculation of Annual Doses to Man from Routine Releases to Reactor Effluents for the Purpose of Evaluating Compliance with 10 CFR Part 50, Appendix I, Regulatory Guide 1.109, Revision 1, Washington, D.C., Oct. 

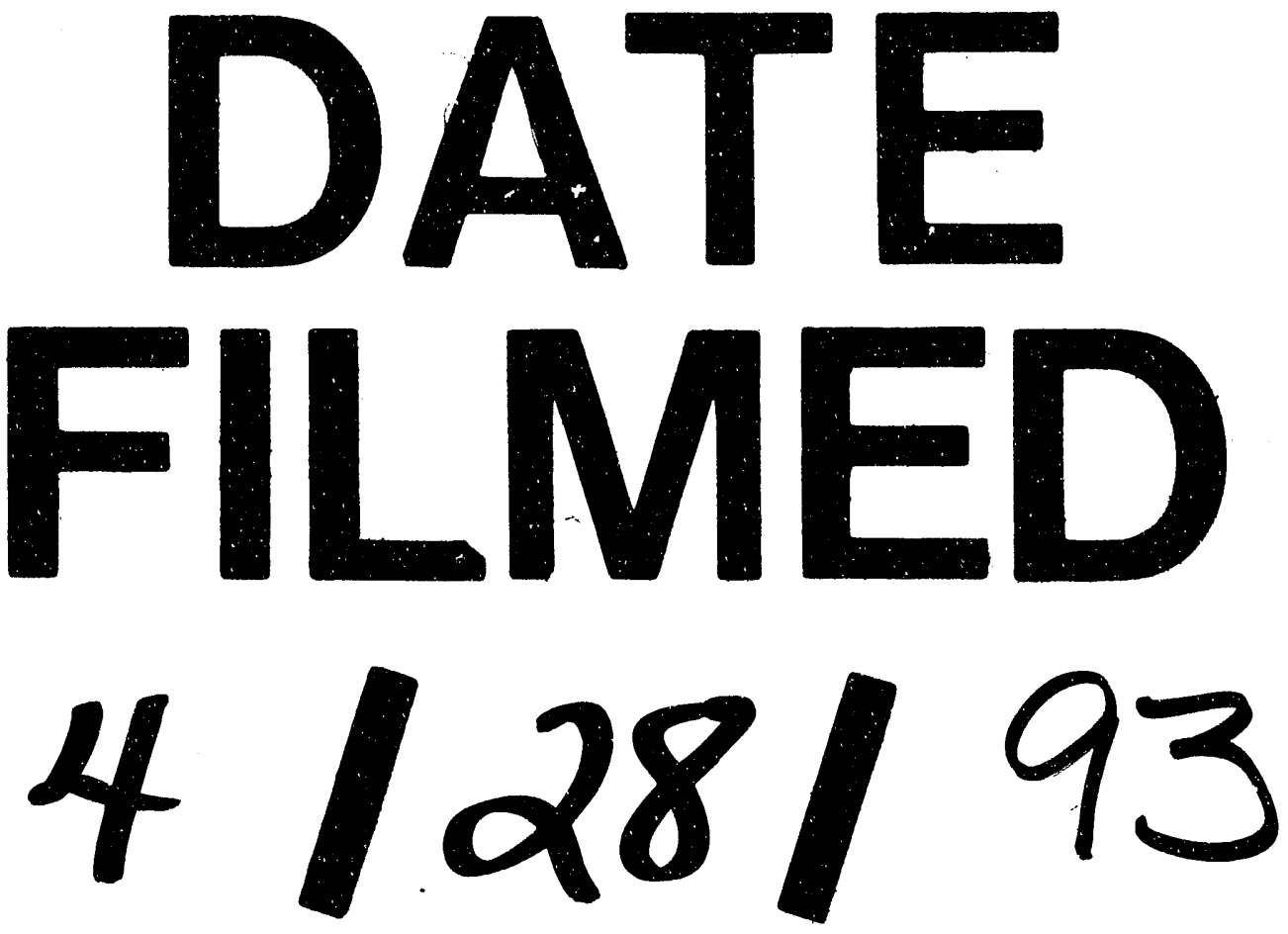